\title{
Educational software for radial power system protection
}

\author{
Nasr-Eddine BOUHENNA \\ Department of Electrical and Electronics Engineering Technology, Higher Colleges of Technology, ADMC - United Arab \\ Emirates.
}

Accepted 24 April, 2013

\begin{abstract}
This paper presents a set of analysis tools for self learning for radial power system protection training (Bouhenna 2010). It automates a radial feeder for an overcurrent protection that would include fault calculations, fuse-fuse co-ordination, current transformer, relay-relay and fuse-relay co-ordination, directional overcurrent protection, ground fault protection, automatic recloser and sectionalizer. The software explains the function of all these protective elements and provides the students with the skills and knowledge necessary to understand and comprehend radial power system protection.
\end{abstract}

Key words: Set of analysis tools, radial power system protection, fault calculations, co-ordination, protective elements.

\section{INTRODUCTION}

Many existing materials cover most of the power system protection topics in a very deep manner which may be difficult for a new learner. Without any support, the student will have difficulties in understanding the topics. Protection coordination is based on devices' operating characteristics where students are able to select and configure protection devices and display their corresponding time-current characteristics. Different scenarios can be applied with different combinations from simple network to advanced coordination schemes. After completing this course, the student will have accumulated enough skills to be able to do the protection design of a complex radial network.

Some previous related work was developed which does not seem to help a new learner to understand the concepts of the coordination in power system protection. Three of them are mentioned as follow: A Matlab /GUI Based Fault Simulation Tool for Power System Education (Koç and Aydoğmu, 2009), (covers only the fault calculations); Computer Assisted Learning in Power System Relaying (Lai, 2002) (does not cover all the different protection schemes, it is suitable for students who already know the basic schemes); Easy Power (ESA, 2009), (delivers a full lineup of powerful Windowsbased electrical software tools for intelligently designing, analyzing, and monitoring electrical power systems, but it does not explain in details how the fault currents have been calculated or how the coordination time interval between two protective devices has been found).

\section{METHODOLOGY}

The software developed in this paper is based on Excel/Visual Basic Applications to allow learning and analysis. This user-friendly educational software presents a comprehensive and systematic description of the concepts and principles of operation and application of protection schemes for radial power system. It covers the calculation of the per-unit and power system faults for small scale systems. It is followed by the basic protection functions of the fuse, current transformer, and different types of protective relays.

Most of the students are quite familiar with the main functions of excel worksheet when they have to enter data and write formulae. A set of tools consisting of multiple choice questions, true or false 
applications, animated circuits, correct or wrong answer applications, and plotting curves make the software really interactive and helps considerably the student to understand the concept of the protective devices.

This software which has been developed contains the essential material to cover the basic concepts of radial power system protection. It is developed in such a way that the students need no extra resources to understand the principles of protection systems. Most of the circuit diagrams in this software are animated and the student is guided through to the correct answer. A variety of networks for different situations are presented in this program to further enhance the understanding of the subject matter.

The software developed is a training model and teaching tool for radial power system protection. The model will serve as supplementary material or training tool for engineers entering this field.

\section{RESULTS AND DISCUSSION}

\section{Software description}

A user-friendly Excel /GUI based software has been developed to present a set of analyses tools for self contained study that will help students / trainees understand the radial protection schemes in electric power systems. It aims to provide the student with enough skills to understand the concept of the main protective devices and their operation in electrical power systems. Definitions and formulae are well presented. For each of the topics below, the student animate the circuit as an introduction to the problem he or she has to solve as it is explained in part 3. The software covers the following topics (Stenven 2010; John and Jan, 2010; Areva and Alstrom, 1995; Keit, 2008; Ron, 2008; Soman, 2008; John and William, 2003).

(a) Per-unit section

(b) Fault section

(c) Fuse section

(d) Current Transformers section

(e) Relay Part (1) section

(f) Relay Part (2) section

The menu in (Figure 1) represents all the applications which have been developed using Excel/Based Visual Basic Applications. There are six sections which are: PerUnit, Fault, Fuse, Current Transformer, Relay Part 1 and Relay Part 2.

There are a total of 44 applications. The student can switch from one section to another by just clicking on the button 'Back to Menu'.

The applications of the six topics are detailed in the Table 1.

Five assessment models have been developed to cover all the chapters which have been detailed as follows:

(i) Model 1: Multiple Choice Questions

(ii) Model 2: True or False (iii) Model 3: Animated Graphics

(iv) Model 4: Correct or Wrong answer

(v) Model 5: Data Setting

\section{Assessment model of the software}

Five assessment models are made to help the student to understand each application of each topic.

\section{Model 1: Multiple choice questions}

A set of multiple choice questions (Figure 2) is presented to the student to check his or her knowledge after reading the topic related to the fuse from the material given.

\section{Model 2: True or false}

Ten questions are presented to the student (Figure 3). The student has to select either True or False. The response is correct if all the 10 answers are correct.

\section{Model 4: Animated graphics}

An example of a relay-relay coordination as shown in Figure 4 will help the student to understand the coordination between the primary relay and the backup relay when a fault occurs at different locations. The characteristics are displayed for each step that will help the student to understand the importance of the coordination time interval between the two relays.

Operational logic of two-count sectionalizer can be summarized with reference to Figure 5, which illustrates the responses to a transient fault and permanent fault when the fuse saving scheme is used. Step by step the student can display the operation by just clicking on the sequence button. The animated circuits/graphics as an introduction to the application help the student to understand better the topic so that he or she will not have problem answering the questions presented just below the graphic.

The animated graphic in Figure 6a shows the operation of the directional relays R3 and R5. The animated circuit shows the two scenarios, one with the directional relays and the other one without the directional relays. When there is a fault at F2, only the directional relay R3 sees the fault and disconnects the feeder 1 . The load is still getting energy from feeder 2 as shown in Figure $6 \mathrm{~b}$. All the information during this short time is displayed on the screen. The operation of the directional relays is shown at three different locations.

\section{Model 3: Correct or wrong answer}

A set of applications to be solved is presented to the 


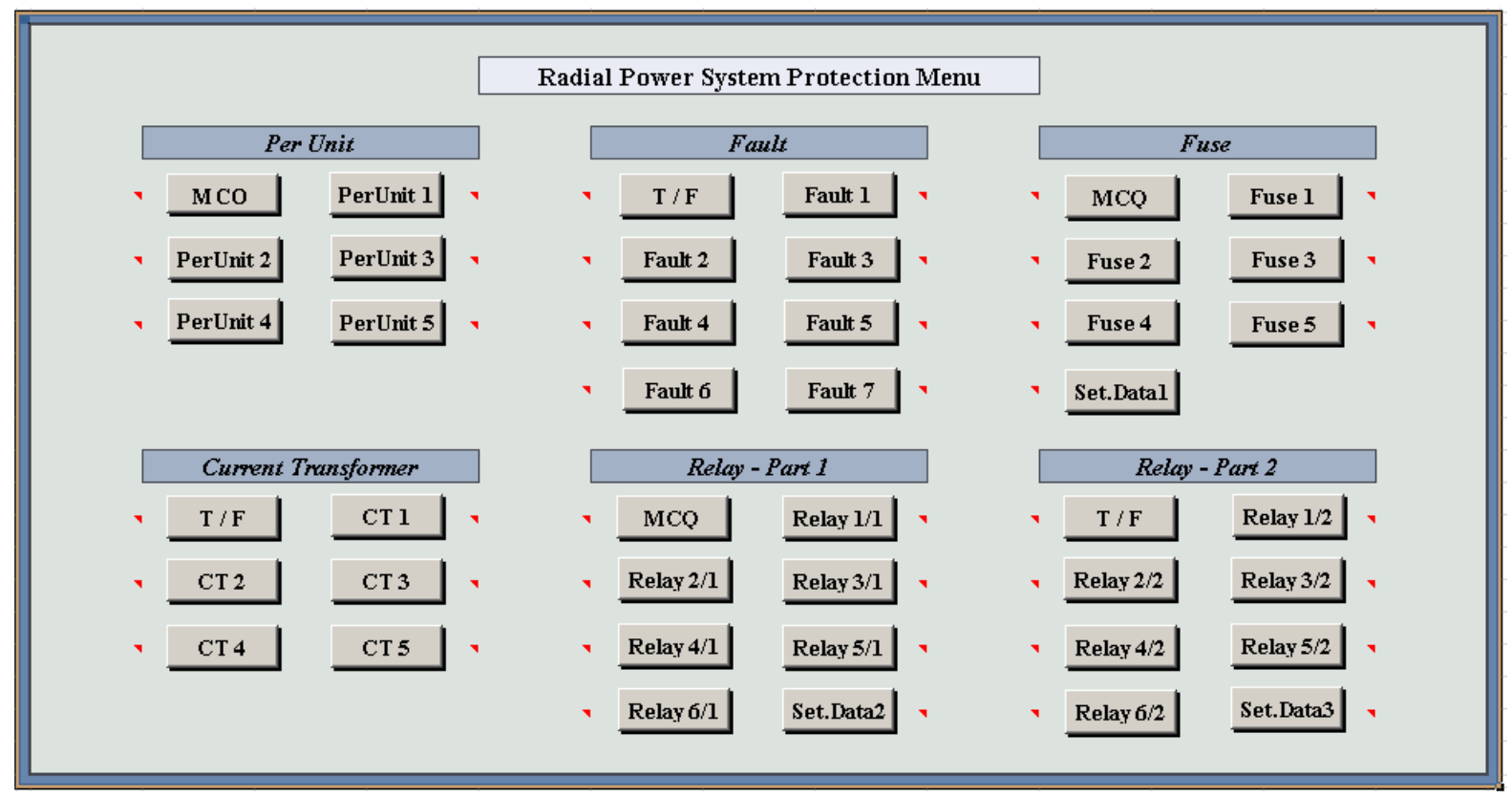

Figure 1. Radial power system protection menu.

Table 1. Software content.

\section{(a) Per-unit section:}

. Multi-choice questions

. Use of per-unit

. Transformer per-unit calculations

. Per-unit value of a . transmission line

. Transmission system per-unit calculations

. Per-unit impedances in single phase transformer circuits

\section{(d) Current transformer section:}

. True / false

. Current transformer - relay

. Current transformer performance calculation

. Current transformer saturation

. Multi-ratio current transformer performance

. Effect of saturation on current transformer

\section{(b) Fault section:}

. True / false

. Balanced system 3-phase fault (1)

. Balanced system 3-phase fault (2)

. Symmetrical components of unsymmetrical phasors

. Operator "a" and unbalanced system

. Various types of short-circuit faults

. Line - to - line ground fault

. Line - to - line fault and line - to - line - to - ground fault

\section{(e) Relay part (1) section:}

. Multi-choice questions

. Relay operation

. Relay - relay coordination

Setting - up the relay

. Getting familiarized with setting data (2)

. Fault calculations and relay - relay coordination

. Plug setting multiplier and phase relay coordination

. Setting data (2): Relay characteristics display

\section{(c) Fuse section:}

. Multi-choice questions

. Clearing - melting time

. Fuse selectivity ratio

. Fuse - fuse coordination

. Fuse type selection

. Low voltage fault - case study

Setting data (1): Fuse characteristics display

\section{(f) Relay part (2) section:}

. True / false

. Fuse - MCCB - relay coordination

. Directional relay coordination

. Earth fault relay coordination

. Current transformer - burden relay

. Auto - reclosing relay selection

. Recloser - sectionalizer operation

. Recloser - sectionalizer - fuse coordination

. Setting data (3): fuse - relay characteristics display

.Challenge problem. 


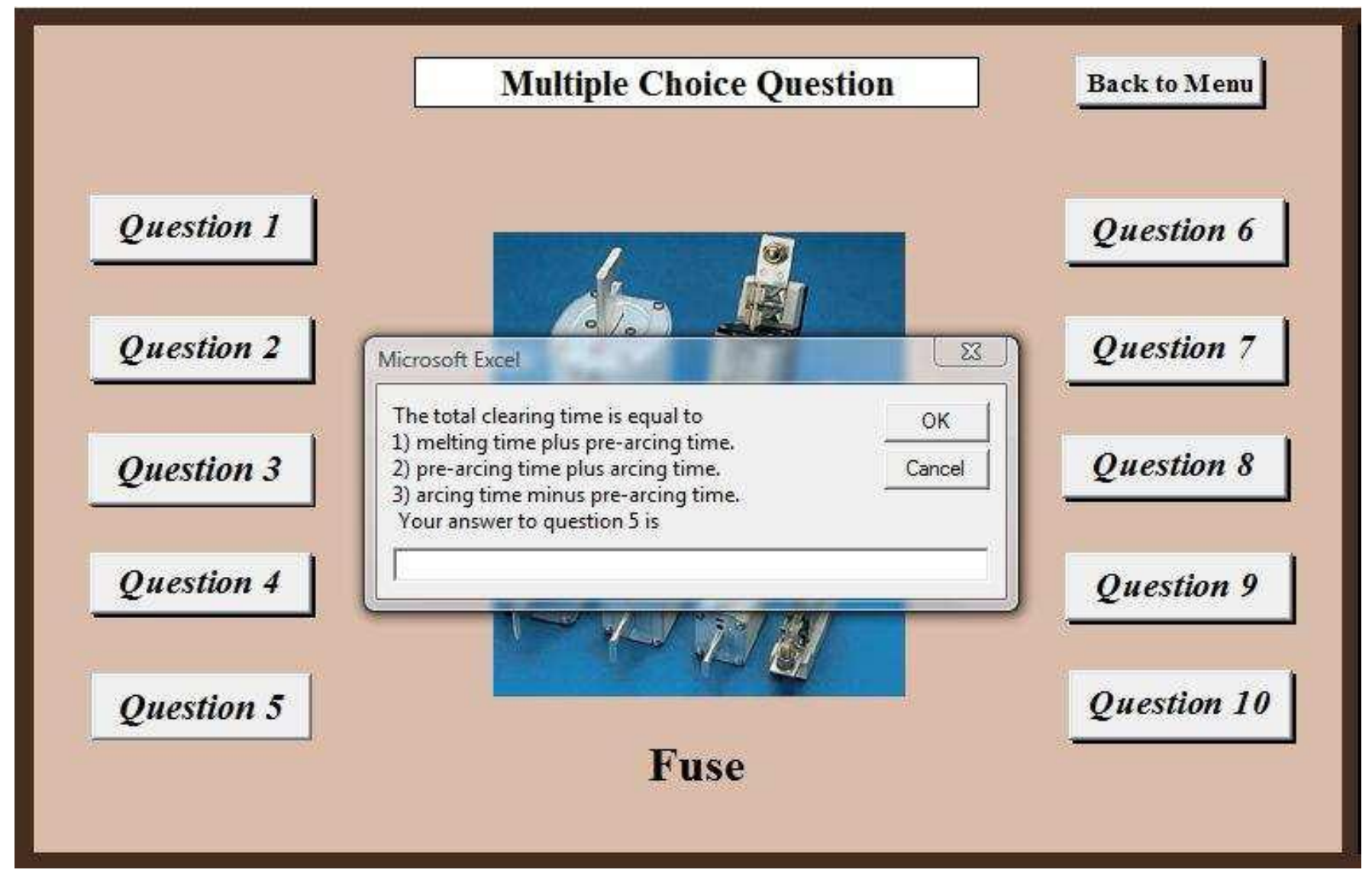

Figure 2. Fuse multiple choice questions.

student for each topic as it is shown in Figure 7. After an animated introduction, the student has to solve a problem by entering values or selecting good answers from the selection box. The application question and some notes can be displayed on the screen by just clicking on the appropriate button. Correct or wrong answer can be checked after entering each answer.

\section{Model 5: Setting data}

It's important to display the characteristics of the protective devices such as fuse and relay so that the coordination becomes easier to check.

A total of 6 data tables are presented to the student, which means 6 different curves to plot. Once the data of the protective fuse is entered in the proper locations (white cells) as shown in the data table of Figure 8, a simple click on "Do Calcs" button will display the time current characteristics of the protective device and the fault current line will show the corresponding tripping time response. Three setting data are made, one for fuse (setting data 1), one for relay (setting data 2) and the third one is for fuse-relay combined together (setting data $3)$.

\section{Conclusion}

Protection system's main function is to quickly clear faults from the power system by minimizing the time for which a fault remains in the circuit. This helps enhance people's safety and minimize equipment damage while maintaining power system stability. Protection of power systems requires an understanding of system faults and their detection, and the safe isolation of the faulted device from the system.

This educational software will explain the function of all the protective elements in detail and provide the students with the skills and knowledge necessary to calculate fault currents, coordinate fuses, select and coordinate relays and associated instrument transformers.

The software covers the calculation of the balanced and unbalanced faults using the symmetrical components. The grading between two fuses has been detailed so that the grading between the downstream and upstream fuses will generally be achieved if the current rating ratio between them is equal or greater than two or by using the $\mathrm{I}^{2} \mathrm{t}$ Let-through Energy condition. The grading between two relays has been applied in many applications using the formulae of the four standard current time characteristics - standard inverse (SI), very 


\begin{tabular}{|l|}
\hline Question 1 \\
\begin{tabular}{|l|l|}
\hline Voltage transformers or VTs and Current transformers or CTs reduce real power quantities to quantities \\
measurable by instruments and relays. They insulate instruments and personnel from very high voltage
\end{tabular} \\
\hline Question 2: \\
\hline The output impedance ("burden") must be high so that the saturation will not occur. \\
\hline
\end{tabular}

Question 3:

The CT can only supply a certain secondary voltage before iron saturates - design and impedance of relay, meters etc is very important.
$\bigcirc$ TRUE
FALSE $\bigcirc$

\section{Question 4:}

There are two types of CT. Measuring CTs type "M" and Protection CTs type "P". Measuring CTs designed to operate at fault current and Protection CTs designed to operate at rated current.

$$
\text { TRUE FALSE } \bigcirc
$$

\section{Question 8:}

A knee-point voltage Ekn is defined to be the point where a $10 \%$ increase in exitation voltage results in a $50 \%$ increase in exitation current and it is defined by Ekn $=\mathrm{Kd} x($ Rct + Rlead + Rburden) $\mathrm{x}$ Isn.

$$
\text { T TRUE FALSE } 0
$$

\section{Question 9:}

A fault current of $1 \mathrm{kA}$ is detected by a CT of $500: 5$. The secondary current would be equal to $10 \mathrm{~A}$.
- TRUE
FALSE
$\circ$

\section{Question 10:}

The letter $\mathrm{C}$ stands for "calculated" which means the CT accuracy can be determined by calculation from given excitation curves. The number after the letter is the specified terminal voltage the CT will maintain without exceeding the specified $10 \%$ ratio error with 10 times rated current in the secondary.
T TRUE
FALSE
$\bigcirc$

Figure 3. Current transformer true or false. 


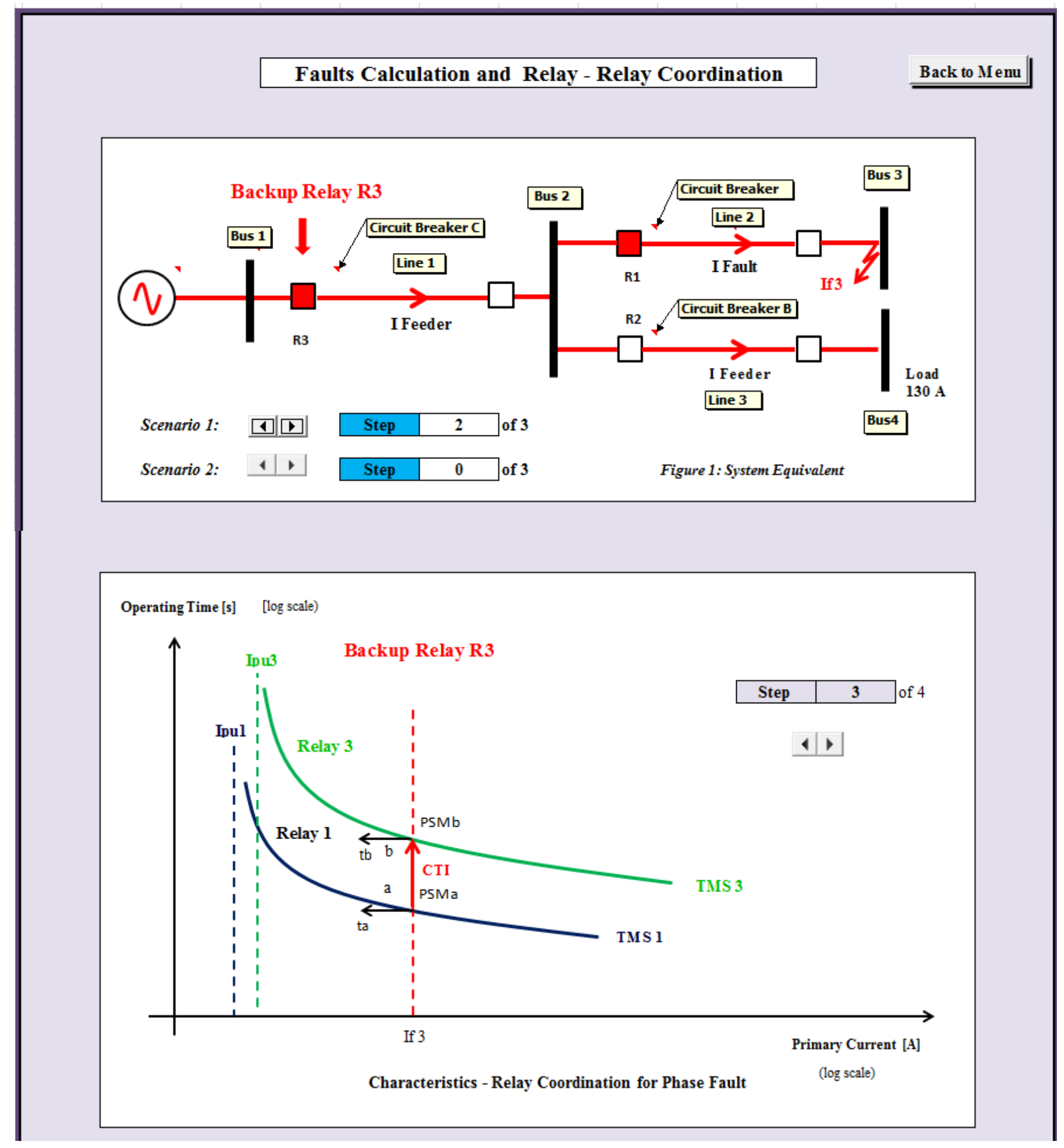

Figure 4. Relay - relay coordination (animated graphic)

inverse (VI), extremely inverse (EI) and long-time inverse (LTI). The curves can be displayed to verify if the coordination is achieved.

The student will also learn how to adjust the setting of the relays using the standard operating characteristics so that the relays closest to the fault will operate and clear the fault faster than the backup devices and also how to successfully coordinate the fuses and relays with both 
Notes:
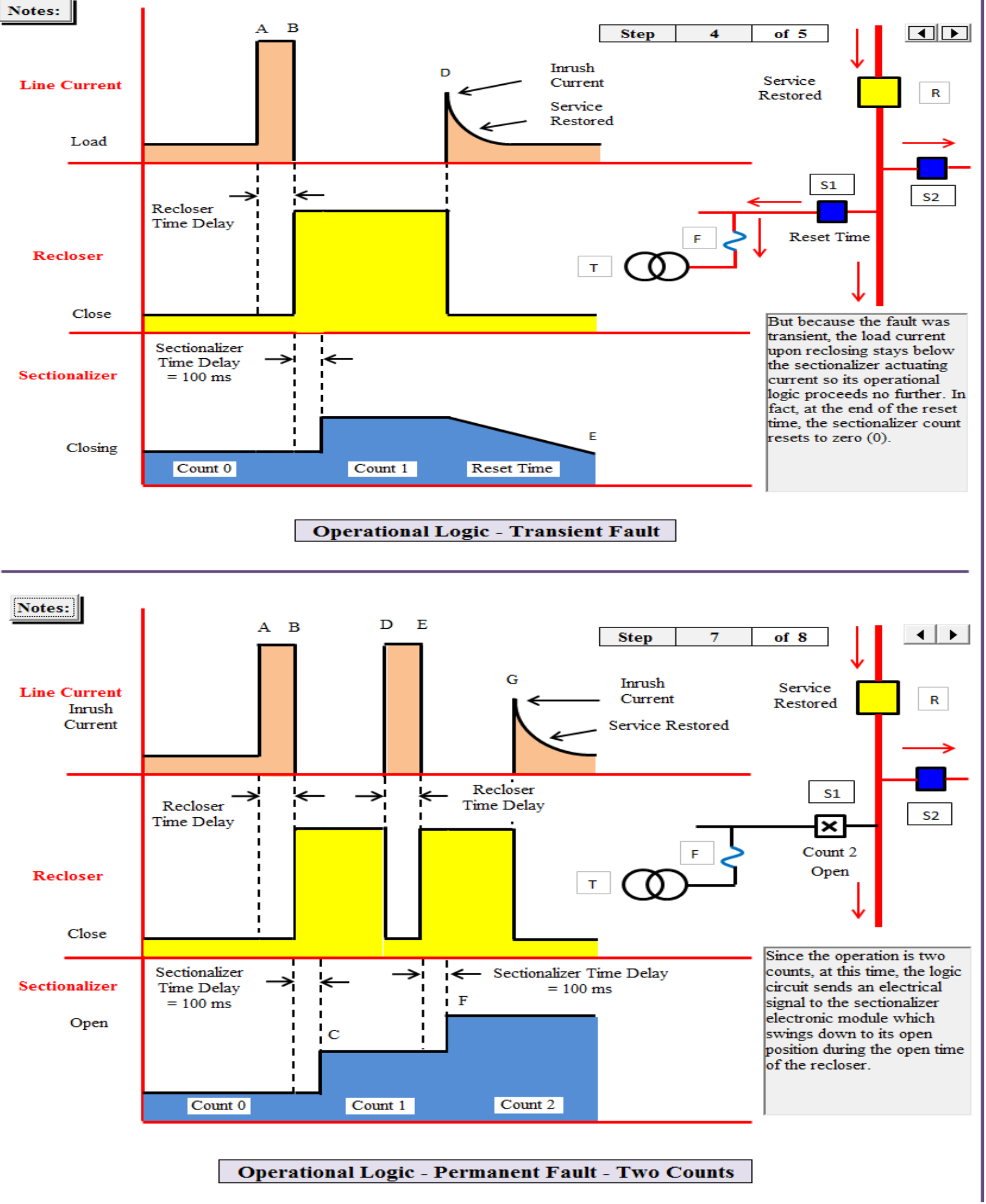

Figure 5. Recloser - sectionalizer operation (animated graphics) 


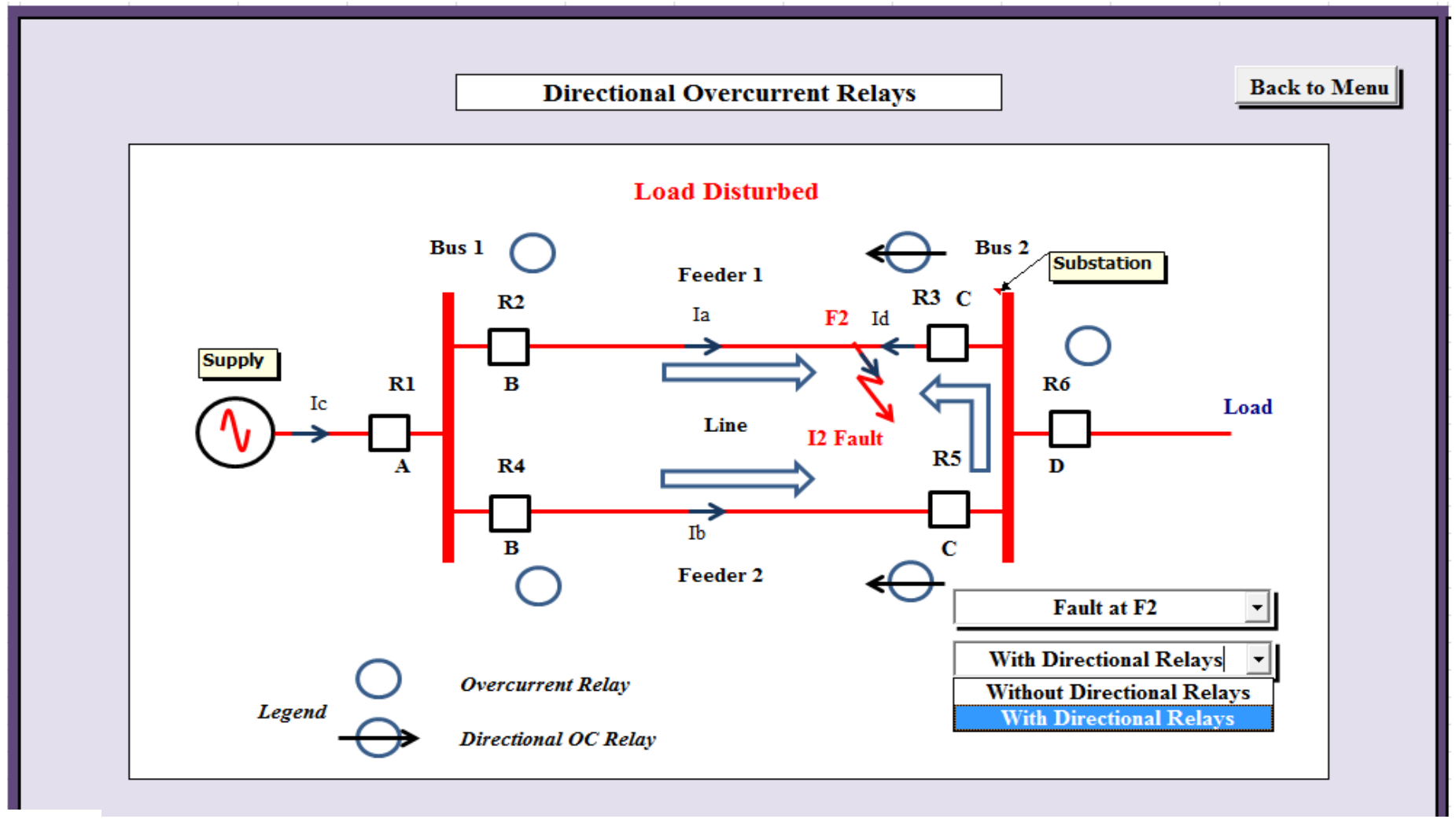

(A)

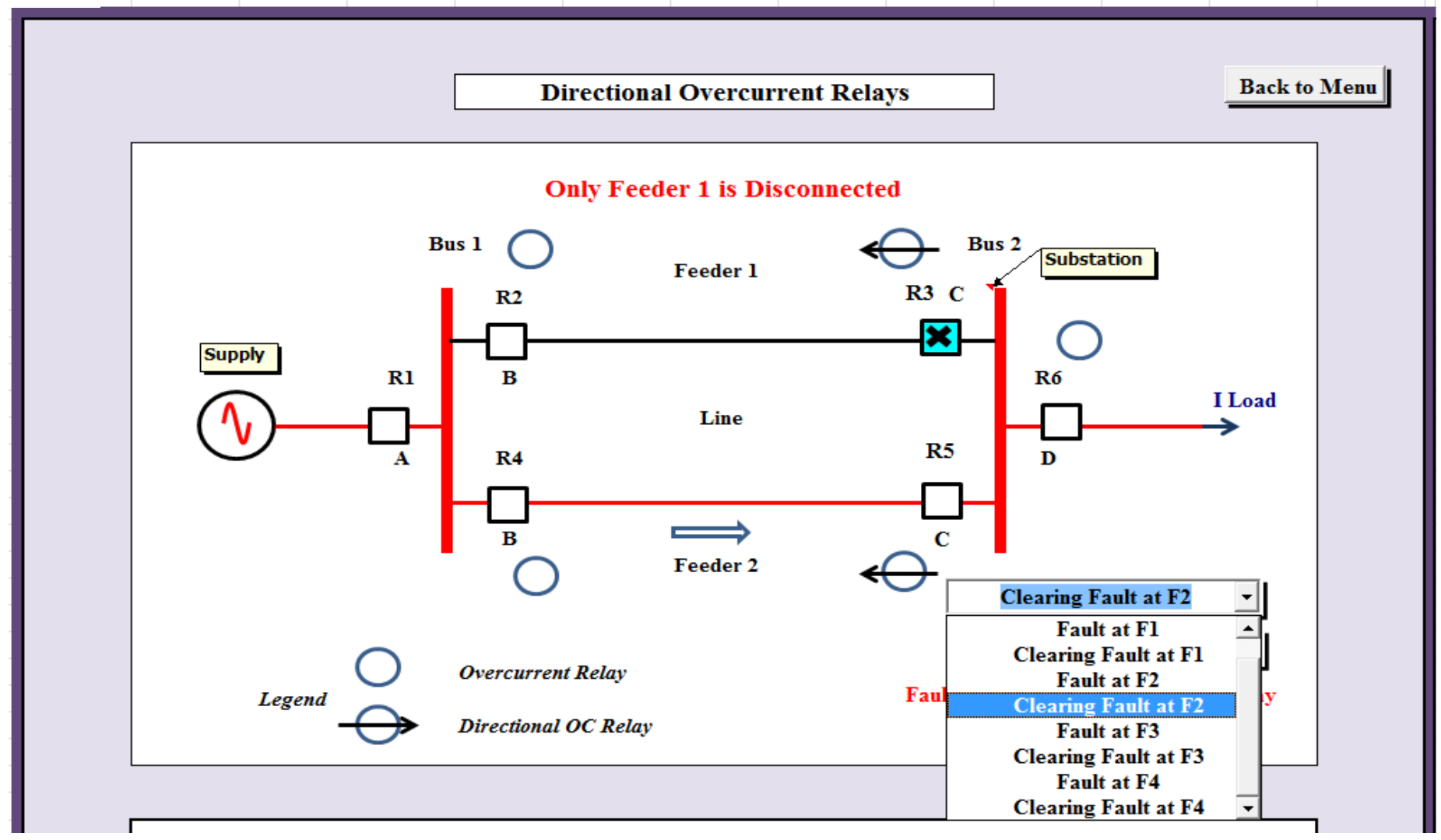

(B)

Figure 6. (a) Operation of directional relays at fault location F2. (b) Clearing the fault by the directional relay R3 at fault location F2. 


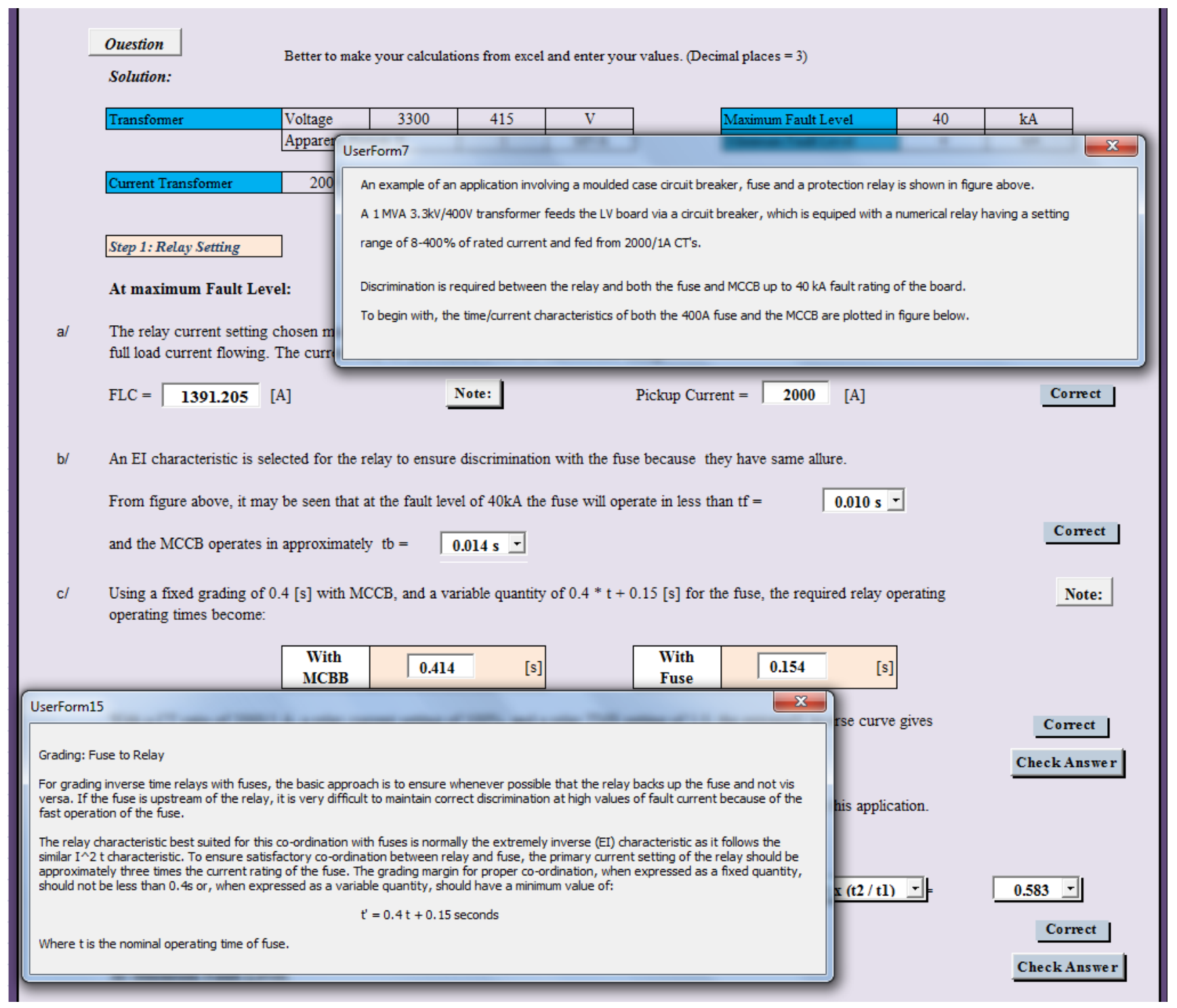

Figure 7. Fuse - MCCB - relay coordination (correct or wrong answer).

source-side and load-side protective devices, as well as the coordination of the other types of relays such as directional relays, earth fault relays, and recloser relays.

The software can be extended to cover distance protection, differential protection, and pilot relaying protection. With more complex systems, it is necessary to detect the point of fault precisely and trip only those sections affected by the fault while the rest of the system can continue to function normally. This requirement necessitates different forms of relaying apart from the simple current sensing relays. Equipment such as generators, transformers and motors also need special forms of protection characterized by their design and operating principles.

The software will become complete and will then emphasize advanced protection schemes required for practical systems experienced in industrial plants, distribution, transmission and generation systems.

In summary, at the end of the course, the student/trainee will have a solid background in protection concepts to apply them to protective devices for radial protection schemes in electric power systems. After completing all the topics, a challenge problem is given the student to check his or her knowledge on a radial network system.

\section{ACKNOWLEDGMENT}

Author would like to sincerely thank and acknowledge the following people for their assistance, guidance and support throughout the duration of this project: firstly, the 


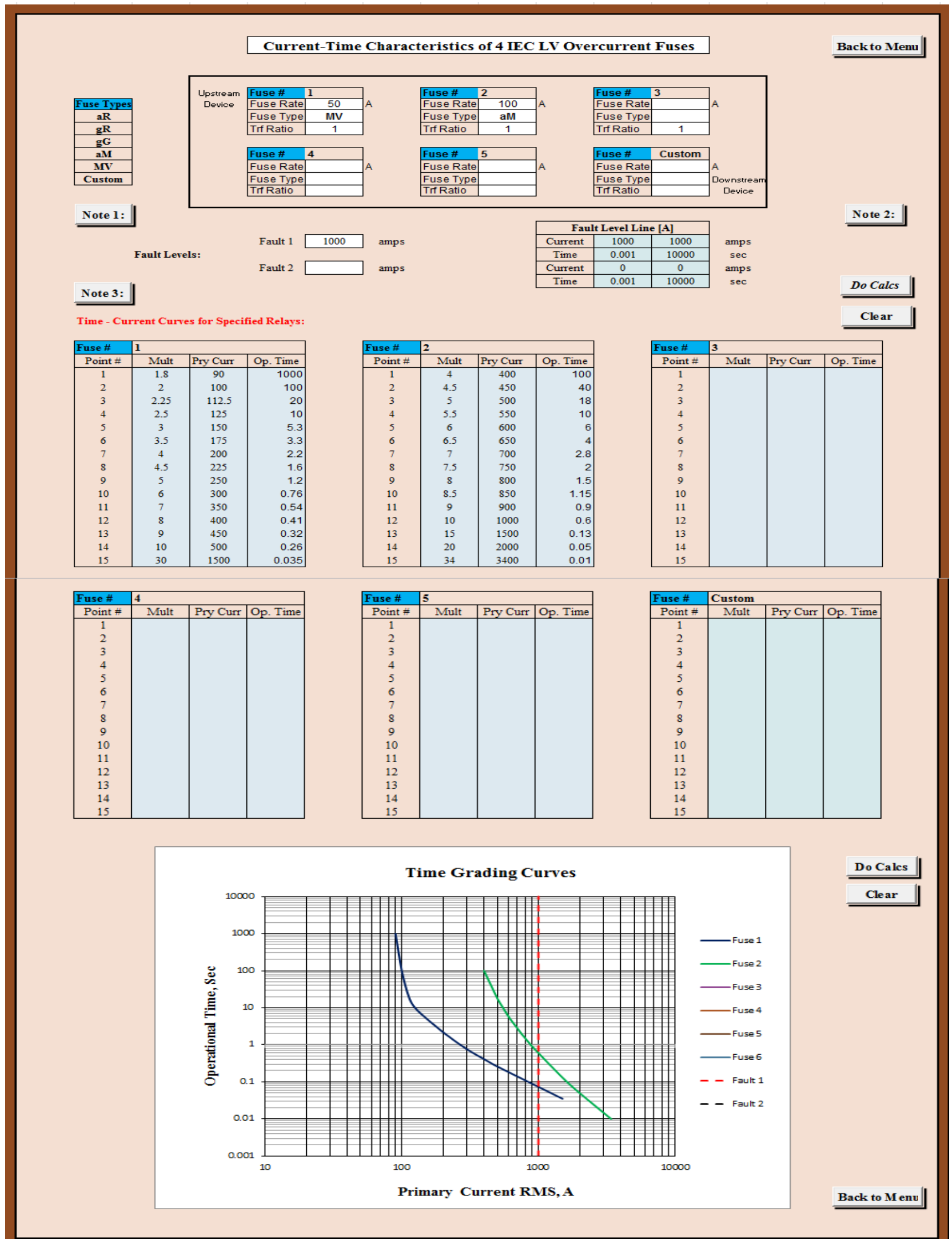

Figure 8. Fuse characteristics display (setting data 1). 
supervisor Dr Tony Ahfock, Deputy Head (Electrical, Electronic and Computer Engineering, University of Southern Queensland, Toowoomba Campus). This study would not have been possible without his support and advice. His wide knowledge has been of great value to the development of this study. It has been a pleasure working with him. The author is also very grateful to $\mathrm{Dr}$ Shakib Farhat, Dean of Engineering at Abu Dhabi Men's College for the continued support and interest in this project. The support and encouragement of Electronic Department Team, his immediate family, and friends are gratefully acknowledged.

\section{REFERENCES}

Areva TD, Alstrom P (1995). Network Protection and Automation Guide. 1 st edn.

Bouhenna N (2010). Radial Power System Protection Training Software. Project and Dissertation Submitted in Fulfillment of the Requirements for the degree of Master of Engineering Technology (Power System Engineering) at The University of Southern Queensland, Faculty of Engineering and Surveying, Australia.
ESA (2009). EasyPower, viewed on Mars 2009.

John JG, William DS (2003). Power System Analysis. Tata McGraw-Hill, New Delhi.

John W, Jan KP (2007). Microsoft Office Excel 2007 VBA Programming for DUMMIES. Willey Publishing, Inc., USA.

Keit M (2008). Power System Protection, Electrical and Information Engineering, viewed Apr. 08.

Koç S, Aydoğmu Z (2009). A Matlab /GUI Based Fault Simulation Tool for Power System Education, University Technical Education Faculty.

Lai LL (2002). Computer Assisted Learning in Power System Relaying. IEEE Education Society.

Ron S (2008). Electrical Plant. University of Southern Queensland, Toowoomba.

Soman SA (2008). Power System Protection, Department of Electrical Engineering IIT Bombay,

Stenven CC (2010). Introduction to VBA for Excel. 2nd edn. Pearson Higher Education, New Jersey. 